\title{
Development and usability of myfood24: an online 24-hour dietary assessment tool
}

\author{
M.C. Carter ${ }^{1}$, S.A. Albar ${ }^{1}$, M.A. Morris ${ }^{1}$, U.Z. Mulla ${ }^{2}$, N. Hancock ${ }^{1}$, \\ C.E.L. Evans ${ }^{1}$, N. Alwan ${ }^{1}$, D.C. Greenwood ${ }^{3}$, L.J. Hardie ${ }^{4}$, G.S. Frost ${ }^{5}$, P.A. Wark ${ }^{2}$ and \\ J.E. Cade ${ }^{1}$ on behalf of the Myfood24 Consortium \\ ${ }^{1}$ Nutritional Epidemiology Group, School of Food Science \& Nutrition, University of Leeds, ${ }^{2}$ Global eHealth Unit, \\ Department of Primary Care and Public Health, London School of Public Health, Imperial College London, ${ }^{3}$ Division \\ of Biostatistics, ${ }^{4}$ Molecular Epidemiology Unit, University of Leeds and ${ }^{5}$ Nutrition and Dietetic Research Group, \\ Department of Investigative Medicine, Hammersmith Hospital, Imperial College London, UK
}

Dietary assessment in large scale population studies presents challenges to the researcher. Traditional methods of dietary assessment are paper-based and often require manual nutritional coding which can be time-consuming and costly. An on-line dietary assessment system could potentially reduce researcher burden by automatically coding food records (1). The aim of this research was to develop an online dietary assessment tool for use in the UK.

We report here on the acceptability and usability of myfood 24 (measure your food on one day) during its multistage and iterative development. Focus groups were conducted with three age groups, adolescents $(11-18 \mathrm{yrs})(\mathrm{n}=28)$, adults $(19-64 \mathrm{yrs})(\mathrm{n}=24)$ and older adults $(\geq 65$ years) $(n=5)$ to inform development of the tool. To ensure the system is intuitive and easy to use, a new food composition database was developed for the tool, and the database search function was refined. The new food database developed for myfood24 contains $\sim 45,000$ UK branded and generic foods with their associated pack and portion sizes. This compares to only $\sim 3,500$ generic food items in the standard British food composition tables (2). Usability was assessed with the system usability scale (SUS) scores (measured on a scale of 0-100) (3). This was undertaken with both the beta version and subsequently the live version (adolescents $n=70$, adults $n=20$, older adults $n=4$ ).

Focus groups found that for all age groups, they preferred images to aid portion size estimation and a clean design with no 'popups'. Adolescents were unwilling to spend more than 15 minutes completing the tool, though older adults were happy to spend longer than this. All groups expressed a desire for feedback on their nutritional intake. The self-rated technology confidence score of those who tested the tool was high with median $8(\mathrm{IQR}=2)$ out of a maximum of 10 for adults. Following improvements to the beta version, the live version resulted in good rating scores for the adolescents (median $\mathrm{SUS}=73, \mathrm{IQR}=22$ ) and adults (median $\mathrm{SUS}=80, \mathrm{IQR}=25$ ).

myfood 24 is the first online 24 hour dietary recall tool for use with different age groups in the UK. Usability testing indicates that myfood 24 is suitable for use in UK adolescents and adults. To try a demonstration version of the tool please visit: https://www. myfood24.org/login.

This study was funded by the Medical Research Council (MRC) (ref: G1100235/1).

1. Schatzkin A, Subar AF, et al. Cancer epidemiology, biomarkers \&prevention, 2009;18(4):1026-

2. McCance RA, Widdowson E.M. Cambridge and Food Standards Agency, London; 2002.

3. Brooke J. Usability evaluation in industry. 1996;189:194. 